DOI: $10.24850 /$ j-tyca-2020-04-11

Notas

\title{
Valoración económica del agua de la presa Solís para uso agrícola
}

\section{Water economic valuation of Solís Dam for agricultural use}

Juan Trujillo-Murillo¹, ORCID: https://orcid.org/0000-0001-9962-9017 Arturo Perales-Salvador ${ }^{2}$, ORCID: https://orcid.org/0000-0002-72254574

${ }^{1}$ Universidad Autónoma Chapingo, Texcoco, Estado de México, México, juan_trujillo1989@hotmail.com

2Universidad Autónoma Chapingo, Texcoco, Estado de México, México, adelin21@hotmail.com

Autor para correspondencia: Juan Trujillo-Murillo, juan_trujillo1989@hotmail.com

\section{Resumen}

El objetivo principal de la investigación fue estimar el valor económico del agua de la presa Solís, ubicada en Acámbaro, Guanajuato, México. El 
método utilizado fue la valoración contingente, mediante la estimación de la disposición a pagar (DAP), a través de una encuesta aplicada a productores agrícolas que habitan en la zona aledaña a la presa Solís y que utilizan el agua de dicha presa para el riego de sus cultivos; se consideraron las variables precio, ingreso, educación, percepción y edad. Se aplicaron 50 cuestionarios para la encuesta, que se llevó a cabo en diciembre de 2017. Los datos se analizaron y procesaron usando un modelo binomial Logi, con el software $N$-Logit. La DAP estimada en el área de estudio fue de $\$ 1 / \mathrm{m}^{3}$. Las variables más importantes fueron precio e ingreso; la edad promedio de los encuestados fue de 36 años, y su escolaridad fue medio superior y superior.

Palabras clave: DAP, modelo Logit-binomial, valoración contingente.

\section{Abstract}

The main objective of this research was to estimate the economic value of the water of the Solís Dam, ubicated in Acámbaro, Guanajuato, Mexico. The valuation method used was the contingent valuation, through the estimation of the willingness to pay (WTP), using applied surveys to agricultural producers who live near to the Solís Dam and use the water from it to irrigate their crops, and were considered the variables: price, income, education, perception and age. A total of 50 surveys were applied on December 2017. The data were analyzed and processed using a Logit binomial model whit the $N$-Logit software. The WTP estimated in the study area was $\$ 1 / \mathrm{m}^{3}$. The most important 
variables were price and income; the average age was 36 years, and the education level were high school and university.

Keywords: WTP, Binomial Logit Model, contingent valuation.

Recibido: $28 / 03 / 2019$

Aceptado: $28 / 10 / 2019$

\section{Introducción}

La problemática mundial del agua obedece a factores multidimensionales, lo que sugiere que las posibles soluciones deben contemplar un amplio espectro de acciones encaminadas a garantizar una solución completa, eficaz y efectiva. El aumento en la demanda de agua para la producción de alimentos disminuye la disponibilidad del líquido para sus usos alternos, lo que la convierte en un recurso escaso. Aunado a esto, la producción de biocombustibles deriva en una mayor presión sobre el recurso. Así, el sector agrícola representa aproximadamente $70 \%$ de todas las extracciones de agua dulce a nien el mundo, y más de $90 \%$ en la mayoría de los países menos desarrollados (WWC, 2015). 
Por tanto, la problemática de la utilización del agua como recurso productivo se encuentra principalmente en el sector primario (agricultura y ganadería), ello implica que los esfuerzos para resolverla deben centrarse en este sector, a través del mejoramiento de los procesos, de acuerdo con la optimización del agua, lo cual incluye el perfeccionamiento de los sistemas de riego en el caso de la agricultura. Para el caso de México, la problemática presentada es similar. Así, en el país, en $2014,76.7 \%$ del agua asignada para uso consuntivo la absorbió el sector agrícola; $14.2 \%$, el sector público, y sólo $4.2 \%$ la usó el sector industrial (Semarnat, 2015).

Las condiciones y niveles de utilización de agua de cada país dependen de los requerimientos que tienen los productos que se producen internamente, por lo que para analizar de manera correcta la situación del recurso agua se debe considerar el tipo de producción al que se dedica su sector primario. En términos generales, México (en su sector primario) es productor de cereales, carne, leche y huevo, los cuales son los productos con mayor demanda de agua durante su proceso de producción. Por tanto, $27 \%$ del agua se destina al cultivo de cereales; $22 \%$, a la carne, y $7 \%$ a la leche. Dentro de estos productos, $1 \mathrm{~kg}$ de carne de vacuno requiere de 15000 litros; $1 \mathrm{~kg}$ de carne de pollo, 6000 litros; $1 \mathrm{~kg}$ de cereales, 1500 litros; 1 kg de cítricos, 1000 litros, y 1 kg de legumbres y tubérculos usa 1000 litros (FAO, 2015).

Así, la producción de biomasa y alimentos a través de la función clorofílica exige grandes cantidades de agua para transformar el $\mathrm{CO}_{2}$ atmosférico en materia orgánica mediante agua de lluvia, o aguas superficiales o subterráneas. La producción de $1 \mathrm{~kg}$ de trigo requiere 1 
$\mathrm{m}^{3}$ de agua y $1 \mathrm{~kg}$ de carne emplea $15 \mathrm{~m}^{3}$. Al agua necesaria para producir un bien o servicio se le llama agua virtual (Madurga, 2005).

En algunos estados de la república existen problemas serios con respecto al uso y disponibilidad del agua, ya sea por su ubicación geográfica (lejanía de costas, ríos y presas) o por los costos que representa su utilización; esto aplica principalmente a zonas del noreste, centro y norte del país, como Baja California; al Valle de México y a los estados con problemas de alta contaminación del vital líquido, como Guanajuato. Para el caso de Guanajuato, la mayor parte de su extensión territorial está dentro de la región Lerma-Santiago-Pacífico, lo que le da una alta disponibilidad de agua con respecto al promedio del país. No obstante, existen problemas que disminuyen drásticamente su disponibilidad real de agua, como el hecho de que su precipitación media anual esté por debajo del promedio nacional y un elevado grado de contaminación de sus ríos.

Los niveles de precipitación son un problema con orígenes geográficos y naturales, por lo que la posibilidad de acción es reducida. Pero la contaminación de sus recursos hídricos es una problemática con causas antrópicas, por lo que la intervención de la sociedad mediante acciones que propicien la disminución de los niveles de contaminación es una opción importante. Más allá de recuperar los recursos hídricos contaminados, es urgente la optimización de los recursos que aún quedan disponibles en condiciones favorables para su uso en la agricultura y en los demás sectores económicos, bajo la premisa de que el empleo desmedido de los recursos restantes puede llevar a una escasez generalizada de agua en la zona. 
El municipio de Acámbaro pertenece al estado de Guanajuato, México; se encuentra a 1849 metros sobre el nivel del mar, a $173 \mathrm{~km}$ de la capital. Sus coordenadas son $100^{\circ} 30^{\prime} 06^{\prime \prime}$ y $101^{\circ} 00^{\prime} 00^{\prime \prime}$ de longitud oeste y $19^{\circ} 55^{\prime} 42^{\prime \prime}$, y $20^{\circ} 12^{\prime} 16^{\prime \prime}$ de latitud norte. Acámbaro colinda al norte con los municipios de Tarimoro y Jerécuaro; al sur, con el estado de Michoacán; al este, con Tarandacuao, y al oeste con Salvatierra (Saucedo, 2015).

En Acámbaro se encuentra una de las presas más importantes a nivel nacional por la dependencia de los productores de los municipios cercanos del agua de la presa para el riego de sus cultivos y demás actividades necesarias para su subsistencia, la Presa Solís (ver Figura $1)$.

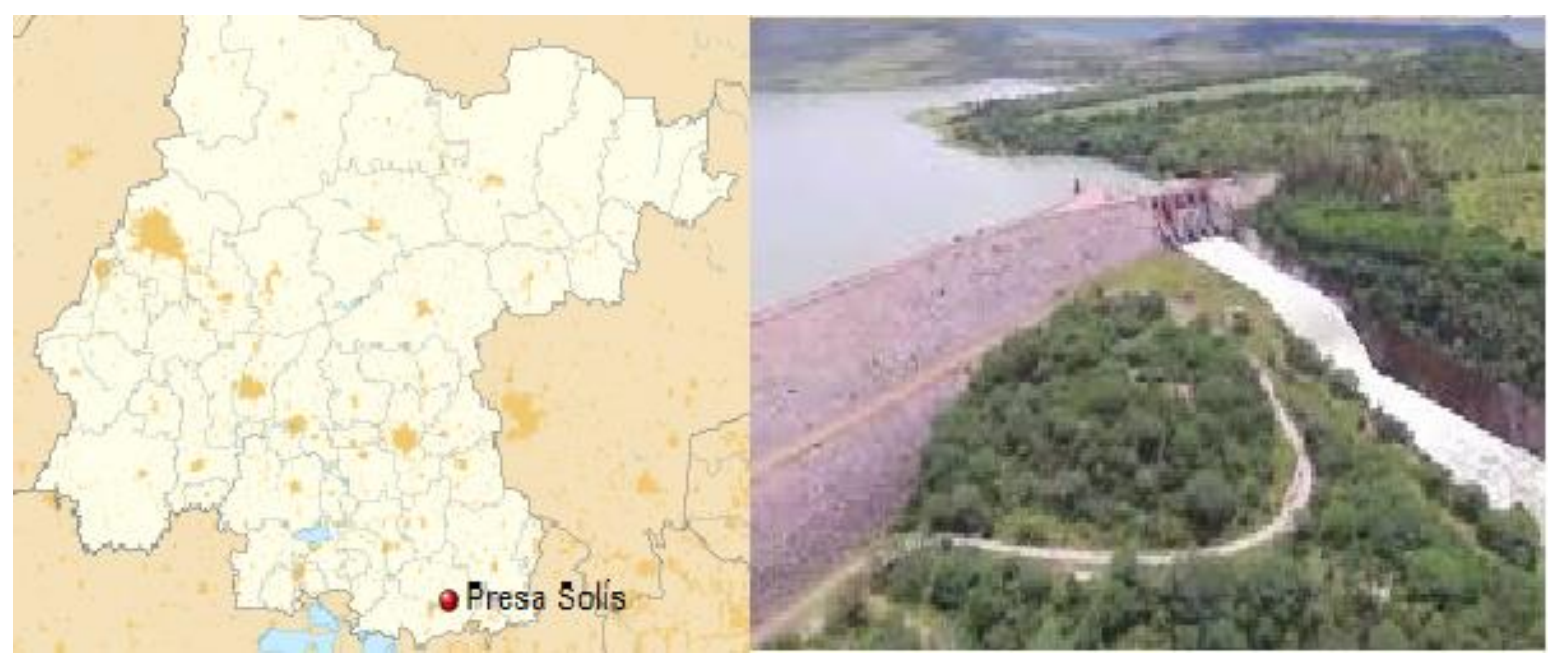

Figura 1. Ubicación de la presa Solís (Conagua, 2016). 
Algunos cuestionamientos que se derivan de la problemática detectada son los siguientes: ¿por qué la valoración económica del agua en la presa Solís repercutiría en su uso racional en el sector agrícola en el municipio de Acámbaro?, de esta pregunta se deriva el objetivo de generar una valoración económica del agua en la zona; también, ¿cómo impactaría la investigación en el bienestar o ingreso de los productores de Acámbaro?, ¿qué estrategias de optimización se podrían implementar? A partir de estas preguntas se genera como objetivo secundario proponer políticas de optimización del agua, lo que lleva al siguiente cuestionamiento referente a ¿cuáles tarifas serían adecuadas para las zonas de estudio? Pregunta para cuya respuesta se utilizará la disposición a pagar por el uso del agua de la presa por parte de los agricultores de la zona; en general, estos cuestionamientos guiaron la presente investigación.

El objetivo principal de la investigación consiste en calcular el valor real del agua como factor productivo mediante la valoración contingente en la presa Solís, en Acámbaro, Guanajuato; al igual que proponer estrategias de optimización del uso del agua en las actividades agrícolas en Acámbaro, Guanajuato. En relación con los objetivos, se presentan las hipótesis de que el valor actual del agua para riego estaría por debajo de su valor real, ya que sólo se toman en cuenta los costos de extracción y/o conducción, y no las externalidades negativas. Por otro lado, se considera la hipótesis de que al pagarse por debajo de su precio real, el agua para riego no se está utilizando de manera óptima, por lo que su disponibilidad futura se torna más limitada e incierta. 


\section{Metodología}

El valor del agua para riego de cultivos agrícolas en la zona de estudio se calculó utilizando el método de valoración contingente (MVC), donde se contrastó el resultado de la valoración teórica con el valor asignado realmente, es decir, su precio de mercado. El trabajo de campo, mediante cuestionarios aplicadas a los productores agrícolas que habitan en Acámbaro, Guanajuato, en la zona aledaña a la presa Solís y que utilizan el agua de dicha presa para el riego de sus cultivos, fue la principal fuente de información para la recopilación de los datos necesarios para la aplicación del método de valoración; se consideró que los productores encuestados utilizan el riego por gravedad, al obtener el agua de la presa, y que los cultivos principales son maíz, frijol y sorgo.

El método de valoración contingente es un método hipotético y directo basado en la información que revelan los agentes económicos involucrados que son encuestados cuando se les pregunta sobre la valoración del bien ambiental. Elemento fundamental de este método es el cuestionario que recoge la información sobre la valoración del bien ambiental (Novoa-Goicochea, 2011). Desde la perspectiva económica, el MVC se basa en la maximización de la utilidad de los encuestados, donde la función de utilidad de los individuos está definida para bienes de mercado y no mercado, configurados por cantidades, precios y otros 
atributos (Avilés et al., 2010). El MVC permite construir un mercado hipotético que simula las transacciones que ocurren en un mercado real para obtener las medidas del cambio de bienestar; mercado en el que los individuos deben expresar su máxima disposición a pagar (DAP) por llevar a cabo una determinada política o actuación (Del Ángel, Rebolledo, Villagómez, \& Zetina, 2009). La idea es cuantificar la DAP promedio como una aproximación del bienestar que refleja las preferencias del usuario. Para este propósito existen tres tipos de formatos: abierto, subasta y referéndum, siendo este último el más utilizado en los estudios de valoración contingente. La característica principal de dicho formato es que sólo se deja al individuo con el problema de decidir si está dispuesto a pagar o no una suma determinada por tener acceso a los beneficios de la política ambiental que se ofrece. En este evento, todas las posibles posturas o propuestas del encuestador se distribuyen de modo aleatorio entre los encuestados (Tudela, 2011).

Para este estudio, las variables endógenas fueron propuestas con base en trabajos científicos de la misma índole, donde se encontró que las variables más representativas en modelos que estiman la DAP son ingreso, nivel educativo, percepción y edad. Algunos de estos trabajos, en el ámbito internacional son el de Aguirre (2015), realizado en Chile, donde determinó que las variables como ingreso, años de educación, estado civil, sexo y conocimiento del tema afectan el resultado de la investigación. En el caso de la variable percepción, Salazar (2001), en su estudio realizado en España sobre la DAP por mejoras ambientales, expone que la información previa sobre el bien objeto de valoración 
influye mediante una relación positiva sobre la DAP. De igual manera, Novoa-Goicochea (2011) evaluó la DAP por áreas protegidas en Perú, obteniendo que las variables más relevantes son precio, edad, ingreso y educación. Más recientemente, con respecto al tema del agua, Tudela (2017) evaluó la DAP por el tratamiento de aguas en Perú, y concluyó que existe una DAP positiva de $\$ 4.30$ por hogar, y las variables que más influyen son tarifa, problemática medioambiental, salud, ingreso y educación. Para el caso de México, estudios recientes sobre la aplicación de la metodología de valoración contingente han arrojado resultados con DAP positiva. Un ejemplo es la investigación de Vázquez (2012), efectuada en Veracruz, la cual encontró que 69\% de los participantes estuvo de acuerdo con pagar un sobreprecio por una mejora en el cultivo de productos agrícolas entre el 10 y $40 \%$ de sobreprecio, donde las variables más importantes son precio, edad, sexo, nivel educativo e ingreso. Otro es el caso de Valdivia, García, López, Hernández, y Rojano (2011), quienes estimaron la DAP por la conservación del río Axtla, ubicado en San Luis Potosí, y concluyeron que existe una DAP positiva promedio de $\$ 57 /$ mes, donde las variables más importantes fueron ingreso familiar, educación y afectación por la contaminación. Otro caso es el de Medina (2014), en el que se afirma que el comportamiento del consumidor está fuertemente afectado por la edad y la preocupación medioambiental. Un caso contundente es el de Silva, Pérez y Návar (2010), donde se obtuvo una DAP positiva por parte del $100 \%$ de los encuestados.

Con respecto a la valoración del agua en México (Avilés et al., 2010), realizada en Baja California, se determinó que los niveles de 
disponibilidad de agua guardan una relación inversa con la DAP, y las variables más relevantes son tarifa, ingreso, educación y disponibilidad del agua. En este mismo sentido, Sandoval et al. (2016) estimaron la DAP por el consumo de agua en la delegación Iztapalapa, Ciudad de México, y obtuvieron una DAP promedio de $\$ 5$ bimestrales, donde la disponibilidad, calidad y precio del agua son las variables más representativas, al igual que las variables socioeconómicas, como edad, escolaridad y sexo. Por su parte, Del Ángel et al. (2009) valoraron el servicio ambiental hidrológico en el sector doméstico de San Andrés Tuxtla, en el estado de Veracruz, y obtuvieron una DAP positiva asociada con ciertas características de la población, en particular ingreso, precio, escolaridad y edad. De igual manera, Jaramillo, Galindo, Bustamante y Cervantes (2013) generaron una valoración económica del agua del río Tlapaneco, en el estado de Guerrero, y tuvieron una DAP positiva, donde la cantidad monetaria que la gente estaría dispuesta a pagar para mejorar la calidad del agua del río fue de $\$ 132.9$ (media), con valores mínimos y máximos de $\$ 40$ y $\$ 300$, una mediana de $\$ 100$ y una desviación estándar de 65.7; las variables más representativas fueron edad, sexo, ingreso, escolaridad y precio del agua. Desde esta óptica, la utilidad, el ingreso, el nivel educativo, la percepción y la edad son las variables contempladas en el modelo. Al considerar la utilidad como la variable dependiente, las variables explicativas determinan el nivel de bienestar generado por el consumo del bien evaluado, en este caso, el agua para riego proveniente de la presa Solís. 
Si el usuario acepta pagar una cantidad de dinero $P$ para mantener el escenario propuesto, debe cumplirse que: $V 1(Q=1, Y-P ; S)-V 0(Q=$ $0, Y ; S)>e_{0}-e_{1}$. Donde los términos $e_{0}$ y $e_{1}$ se asumen como variables aleatorias independientes e idénticamente distribuidas. El cambio de utilidad experimentada por el usuario será igual a la diferencia entre la función de utilidad final menos la inicial; para tener acceso a la utilidad en la situación final definida por el escenario propuesto, se debe pagar cierta cantidad de dinero señalada por el encuestador. Simplificando la notación, se tiene que: $\Delta V=V 1(Q=1, Y-P ; S)-V 0(Q=0, Y ; S)$ yh $=$ $e_{0}-e_{1}$. La respuesta del encuestado (sí/no) es una variable aleatoria. Por lo tanto, la probabilidad de una respuesta positiva por parte del usuario está dada por la siguiente expresión: $\operatorname{Prob}(S I)=\operatorname{Prob}(h \leq$ $\Delta V)=F(\Delta V)$. Donde $F$ es la función de distribución acumulada de $h$. Al elegir una distribución para $h, y$ especificando adecuadamente $V$, los parámetros de la diferencia indicada por $\Delta V$ pueden estimarse con información sobre la cantidad de pago requerida de los individuos, con las respuestas a la pregunta binaria y con la información acerca de las características socioeconómicas de los encuestados (Tudela, 2011).

Con base en la metodología actual utilizada en los estudios recientes, como el de Trujillo, Hernández y Martínez (2019), el modelo explicativo de la investigación se presenta como un modelo de elección binaria, donde la probabilidad de que la DAP sea positiva depende del valor de las variables explicativas:

$$
\begin{aligned}
\operatorname{Prob}(S I) & =\alpha 0+\beta(\text { precio })+\alpha 1 \text { (ingreso })+\alpha 2(\text { edad })+\alpha 3 \text { (percepción) } \\
& +\alpha 4(\text { educación })+\varepsilon t
\end{aligned}
$$


Donde la variable Prob (SÍ) representa la probabilidad de una DAP positiva; en este caso, la disposición a pagar por el uso del agua de la presa Solís. La variable precio expresa el precio simulado por el uso del agua. La variable ingreso se refiere ingreso familiar mensual. La educación está determinada por el máximo grado académico que posee el encuestado. En definitiva, la variable percepción contempla el conocimiento de la situación del agua en la región. Así, la DAP individual por el uso del agua de la presa Solís depende del precio propuesto, nivel de ingreso, educación y edad. En esta investigación, el método utilizado fue la valoración contingente mediante encuestas aplicadas, considerando las variables ingreso, educación, percepción y edad. El objetivo principal del método consiste en establecer un precio que represente el valor del bien en cuestión a través de las cantidades expresadas por los encuestados, considerando que si el mercado del bien existiera, el precio que ellos estarían dispuestos a pagar sería el precio de mercado del bien (Trujillo et al., 2019).

El cuestionario utilizado en la recolección de la información para la aplicación del método de valoración contingente consta de cuatro apartados: el primero aborda el reconocimiento del bien en estudio y de la relación del encuestado con dicho bien; el segundo indaga en la percepción del encuestado sobre el uso del bien en cuestión; el tercero busca obtener la disposición a pagar (o ser compensado) por el uso del bien, y el apartado final es acerca de las características socioeconómicas del encuestado. 
El primer apartado, denominado preguntas de reconocimiento, explora los conocimientos del encuestado acerca del objeto de estudio; es interrogado acerca de la cantidad de agua que utiliza para el riego de sus cultivos, el origen de ésta, sus costos en la zona y la disponibilidad para riego en la región. Se procura conocer su opinión con respecto al correcto uso del agua; es decir, evitar desperdicios, y su grado de preocupación en el tema.

El segundo apartado, denominado preguntas de percepción, centra la atención en el empleo del agua en el proceso de producción por parte del encuestado.

El tercer apartado propone diferentes tarifas a pagar por el uso del agua de la presa Solís para la producción de cultivos agrícolas.

Por último, se exploran las variables socioeconómicas de los encuestados, como nivel de ingreso, escolaridad, sexo, estado civil y edad, para relacionarlos con su DAP. El formato de encuestas se expone en el Anexo 1.

A partir de las respuestas obtenidas en cada uno de los bloques se establece una relación entre la disposición a pagar que expresan los encuestados y sus atributos socioeconómicos. Mediante un modelo Logit-binomial se estima un valor promedio de la DAP por parte de los usuarios del agua. El procedimiento estadístico realizado para llevar a cabo los cálculos y estimaciones de los valores correspondientes a todas las variables que se incorporan en el modelo o que intervienen de alguna manera en él está orientado a extrapolar las respuestas de la muestra poblacional al total de la población. Bajo esta consideración es 
necesario tener en cuenta el nivel de error, no sólo metodológico sino estadístico al momento de interpretar los resultados finales. Se aplicaron 50 encuestas en diciembre de 2017, bajo un esquema de muestreo aleatorio simple con población infinita. Los datos se analizaron y procesaron utilizando un modelo binomial Logit con el software $N$-Logit.

\section{Resultados y discusión}

Se utilizó un código binomial para registrar el nivel de estudios del encuestado y se registró considerando la numeración: 1 = primaria; 2 = secundaria; $3=$ preparatoria; $4=$ licenciatura; $5=$ posgrado. El nivel de ingreso se registró mediante los rangos de ingreso: $1=<\$ 4000 ; 2=$ $\$ 4000-\$ 8000 ; 3=\$ 8000-\$ 12000 ; 4=\$ 12000-\$ 18000 ; 5=\$ 18$ 000-\$25 000; $6=>\$ 25000$. Los límites superiores de cada nivel de ingreso se consideraron como parte del siguiente estrato. La variable edad se registró como una variable lineal ascendente $(18,19,20$, $21, \ldots)$. La categorización de las variables por estratos se registró mediante la utilización de un código numérico, que se presenta en la Tabla 1.

Tabla 1. Código de registro de respuestas. 


\begin{tabular}{|c|l|l|l|c|c|c|}
\hline Código & Educación & Precio $\mathbf{( \$ / \mathbf { m } ^ { 3 } )}$ & Ingreso (\$) & Edad* & Percepción** & DAP \\
\hline 1 & Primaria & 0.5 & $<4000$ & 18 & Amplio & SÍ \\
\hline 2 & Secundaria & 1.0 & $4000-8000$ & 19 & Moderado & NO \\
\hline 3 & Preparatoria & 1.5 & $8000-12000$ & 20 & Bajo & Nulo \\
\hline 4 & Licenciatura & 2.0 & $12000-18000$ & 21 & $\ldots$ & \\
\hline 5 & Posgrado & 2.5 & $>18000$ & $\ldots$ & & \\
\hline
\end{tabular}

* La variable edad por su naturaleza comprende un rango de 18 a 90.

**Conocimiento del tema.

Las variables endógenas del modelo se convirtieron a términos numéricos para poder ingresarlas y analizarlas en el software de procesamiento de datos. Las categorías de cada variable se expresaron mediante estratos, en general cinco, que respondían a los valores contenidos en cada variable y al número de posibles respuestas en el cuestionario. Los resultados de este procedimiento se muestran en la Tabla 2.

Tabla 2. Estadísticas descriptivas.

\begin{tabular}{|c|c|c|c|c|c|}
\hline Variable & Media & Desv. est. & Mínimo & Máximo & Casos \\
\hline Edad & 36.361 & 14.501 & 17 & 74 & 50 \\
\hline Educación & 4.055 & 1.607 & 1 & 5 & 50 \\
\hline Ingreso & 2.5 & 1.855 & 1 & 6 & 50 \\
\hline
\end{tabular}


El 93\% de los productores agrícolas de Acámbaro encuestados fueron hombres y $7 \%$, mujeres, lo que es en parte predecible, pues hoy en día, en el país, el estrato de la población ocupada el sector primario está compuesto principalmente por individuos del sexo masculino. Por la naturaleza de la investigación, la variable sexo es poco representativa, sin embargo fue incluida por los resultados en otras investigaciones, donde se utilizó la DAP aplicada a temas referentes del agua (Flores, González \& De los Santos, 2010), donde 42\% de los involucrados fueron mujeres y el $58 \%$ hombres. Estos datos se aprecian de manera gráfica en la Figura 2.

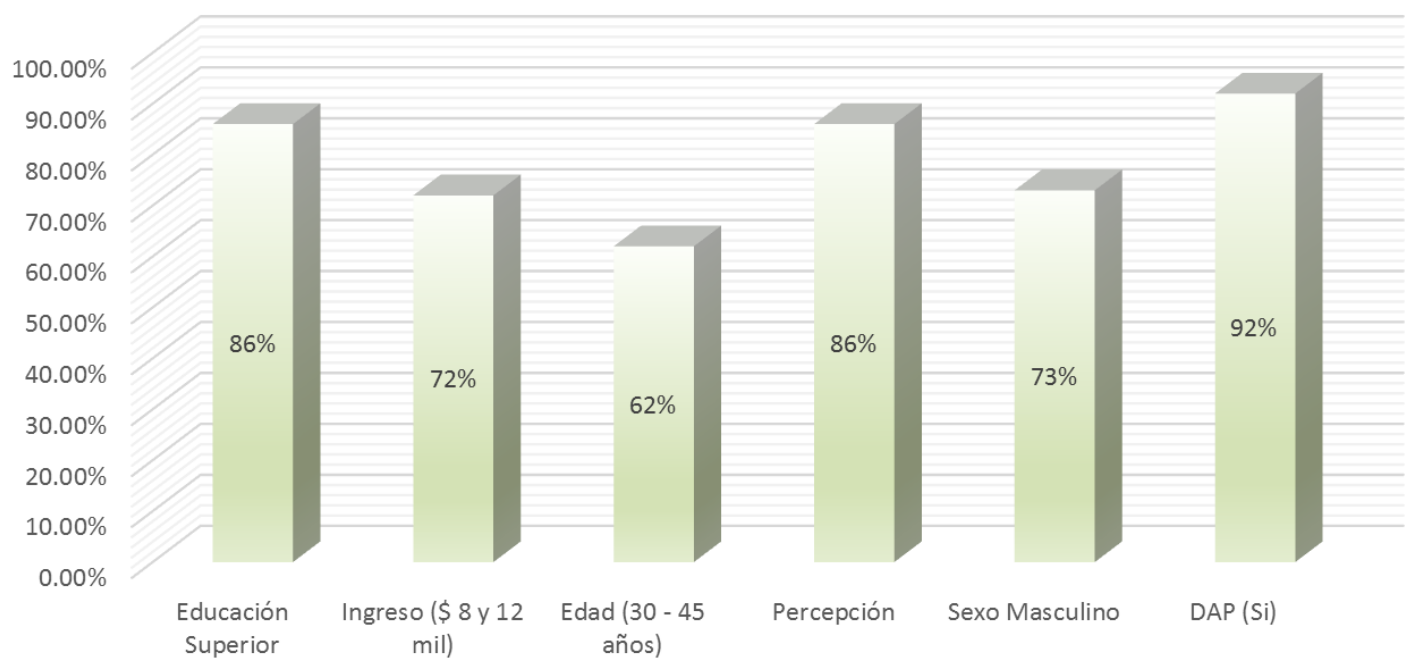

Figura 2. Valores medios de las variables. Elaboración propia.

El promedio de edad de los encuestados fue de 37 años y muestra que los individuos que conforman el subsector de la agricultura en la zona estudiada son adultos jóvenes, a diferencia del promedio nacional; 
esto es muy cercano al resultado obtenido por Flores et al. (2010), donde se obtuvo una edad promedio de 36 años, al igual que el caso de Del Ángel et al. (2009), con un rango de edad de 26 a 35 años. El nivel de estudios promedio de los encuestados fue preparatoria, dicho resultado presenta una aproximación general del nivel educativo de los agricultores de la zona de estudios, lo cual debe tomarse en cuenta al momento de implementar políticas orientadas al sector en el sentido de la forma y los medios para llegar a la población objetivo. Este resultado con respecto al nivel de estudios es parecido al obtenido en el trabajo de Flores et al. (2010), donde la mayoría de los encuestados poseía estudios de nivel medio superior y superior. Finalmente, el ingreso mensual promedio de los encuestados fue de $\$ 8000$ a $\$ 12000$ y se explica por los niveles de costos y productividad en las parcelas, relacionados con los precios medios rurales a los cuales se compra el producto a los campesinos de la zona. Trabajos como el de Flores et al. (2010) reportaron un ingreso promedio a $\$ 11$ 000. Avilés et al. (2010) obtuvieron un ingreso promedio de $\$ 8000$ y Del Ángel et al. (2009) referencian un ingreso promedio de $\$ 7000$. Los resultados de los coeficientes para el modelo se expresan la Tabla 3.

Tabla 3. Modelo propuesto para la DAP.

\begin{tabular}{|l|c|c|c|c|}
\hline \multicolumn{1}{|c|}{ Variable } & Coeficiente & Desv. est. & $\boldsymbol{P}(\mathbf{I Z I}>\mathbf{z})$ & Media de $\boldsymbol{X}$ \\
\hline Constante & -0.459 & 2.562 & 0.800 & \\
\hline Precio & -0.0406 & 0.192 & 0.658 & 1.500 \\
\hline
\end{tabular}




\begin{tabular}{|l|c|c|c|c|}
\hline Edad & 0.058 & 0.026 & 0.061 & 36.361 \\
\hline Educación & 0.319 & 0.282 & 0.500 & 4.055 \\
\hline Ingreso & -0.06 & 0.247 & 0.761 & 2.500 \\
\hline
\end{tabular}

Los resultados de los coeficientes se presentan de la manera esperada, con base en la teoría. En el caso de la variable precio existe una relación inversa entre el precio del agua con la disponibilidad a pagar un monto extra por el recurso; en ese sentido, un precio por debajo del valor real del agua propicia mayor disposición a pagar por el agua. La variable edad muestra una relación positiva entre los años de vida del encuestado y su disposición a pagar por el acceso al agua para regar su tierra; pero no existe una desviación estándar de la edad media lo suficientemente considerable como para extrapolar resultados, pues la mayoría de los encuestados se encontraba alrededor de la mediana edad. El nivel de educativo en los estudios de valoración contingente muestra una relación positiva con respecto a la DAP, lo cual se explica por el nivel de conciencia social y económica que representa la preparación académica y la educación cívica que se fomenta en las escuelas. Los efectos parciales de las variables del modelo sobre la DAP en términos de probabilidad se presentan en la Tabla 4.

Tabla 4. Predicciones del modelo de elección binaria.

\begin{tabular}{|l|c|c|c|}
\hline \multirow{2}{*}{ Valor real } & \multicolumn{2}{|c|}{ Valores predichos } & \multirow{2}{*}{ Total real } \\
\cline { 2 - 3 } & 0 & 1 & \\
\hline
\end{tabular}




\begin{tabular}{|c|c|c|c|}
\hline 0 & $(1.4 \%)^{*}$ & $(18.1 \%)$ & $(19.4 \%)$ \\
\hline 1 & $0(0 \%)$ & $(80.6 \%)^{* *}$ & $(80.6 \%)$ \\
\hline Total & $(1.4 \%)$ & $(98.6 \%)$ & $(100 \%)$ \\
\hline
\end{tabular}

$* *$ Predicciones negativas correctas $=$ total 0 s correctamente predichos.

*Predicciones positivas correctas $=$ total 1 s correctamente predichos.

El aspecto inexacto de las encuestas de opinión y su relación con los valores arrojados por medio de la metodología se presenta con mayor claridad, analizando el grado de predictibilidad del modelo propuesto. El modelo presenta $1.4 \%$ de predicciones negativas correctas, es decir que sólo $1 \%$ de los productores agrícolas encuestados que se esperaba que no estuvieran dispuestas a pagar, en realidad no estuvieron dispuestas a pagar. Por otro lado, $80 \%$ de los productores agrícolas encuestados que, con base en el modelo, se esperaba se mostrarían dispuestas a pagar, lo estuvieron. En esta investigación se obtuvo un elevado nivel de predicción. El total de predicciones correctas por el modelo es de 81 y $19 \%$ de errores en las predicciones del modelo. El nivel de predicción del modelo para el conjunto de variables es aceptable. La DAP estimada en la presa Solís, ubicada en Acámbaro, Guanajuato, fue de $\$ 1 / \mathrm{m}^{3}$ (ver Tabla 5).

Tabla 5. Estadísticas descriptivas de la DAP.

\begin{tabular}{|l|l|l|l|l|l|}
\hline Variable & Media & Desv. estándar & Mínimo & Máximo & Casos \\
\hline
\end{tabular}




\begin{tabular}{|l|l|l|l|l|l|}
\hline DAP & 0.999 & 0.010 & 0.5 & 1.5 & 50 \\
\hline
\end{tabular}

Existe una disposición a pagar media de $\$ 1 / \mathrm{m}^{3}$. Es decir, los productores agrícolas que utilizan el agua de la presa para el regadío de sus cultivos están dispuestas a pagar $\$ 1$ más arriba del precio que hoy en día pagan por metro cúbico de agua. Esto representa la predicción de las hipótesis planteadas, donde los productores agrícolas encuestados presentan una DAP positiva, sugiriendo que el valor actual del agua se encuentra por debajo de su valor real, lo que condiciona la optimización de su uso, lo cual va en concordancia con lo encontrado por Avilés et al. (2010) en el acuífero de La Paz Baja California; por Valdivia et al. (2011) en el río Axtla en San Luis Potosí, y por Sandoval et al. (2016) en Iztapalapa, Ciudad de México.

\section{Conclusiones}

El porcentaje de encuestados que revelaron una DAP positiva fue mayor que el de aquellos que expresaron no estar dispuestos a pagar un monto adicional por el uso del agua de la presa Solís; una DAP positiva por el uso del agua concuerda con los resultados de las investigaciones existentes sobre el tema. En este sentido, el valor real del agua se 
encuentra por debajo de dicho valor, y se concluye que existe un uso irracional del recurso. Esta cuestión cumple el objetivo primordial de la investigación y, a su vez, responde la pregunta planteada al inicio, de la cual surge tal objetivo referente a ¿por qué la valoración económica del agua en la presa Solís repercutiría en su uso racional en el sector agrícola en el municipio de Acámbaro? La valoración expone el consumo irracional del agua y permite proponer estrategias aunadas a vías de acción para racionalizar su uso, orientadas a optimizar la administración del recurso.

El segundo objetivo de la investigación, que habla sobre proponer estrategias de optimización del uso del agua en las actividades agrícolas de la zona de estudio, se cumple a través de la respuesta a las preguntas planteadas que abordan la problemática referente a ¿cómo impactaría la investigación en el bienestar o ingreso de los productores de Acámbaro?, y ¿qué estrategias de optimización se podrían implementar? Además de ¿cuáles tarifas serían adecuadas para las zonas de estudio? Para responder al primer cuestionamiento se retoma el hecho de que el aprovechamiento óptimo del agua permitiría disminuir costos e incrementar ganancias en los sistemas productivos, toda vez que la utilización no óptima conlleva una disminución del bienestar máximo que podrían alcanzar los productores; en otras palabras, utilizarla de manera selecta, considerando su valor real, permitiría incrementar el bienestar de todos los agentes económicos involucrados, lo que responde la primera pregunta.

Para contestar la segunda y tercera pregunta se recurre al hecho de que, cumpliendo con el objetivo principal del estudio, se encontró 
que la DAP estimada en el área de estudio fue de $\$ 1 / \mathrm{m}^{3}$, y con base en estos resultados se propone establecer una tarifa de cuota fija que oscile entre $\$ 1$ y $\$ 2 / \mathrm{m}^{3}$ extra en relación con el costo actual del agua, por el uso del agua de la presa Solís para uso agrícola. Desde esta perspectiva, si se considera que la DAP en general fue positiva, implica que los encuestados en su mayoría ven como una necesidad hacer un uso más racional del agua, en este sentido, una propuesta de optimización es implementar sistemas de riego, como el riego por goteo, considerando que ya existe una disposición a optimizar su uso.

Otra propuesta a considerar es la concientización y educación en relación con los medios y vías para la correcta utilización del agua; esto se desprende del hecho que la variable educación mostró tener una relación positiva y estadísticamente significativa en cuanto a la DAP, por lo que se posee una predisposición a la preocupación medioambiental. Estas estrategias permiten cumplir el segundo objetivo general de la investigación y dan respuesta a las preguntas generadas de la problemática que aborda este estudio.

Considerando el elevado porcentaje de encuestados del sexo masculino, con respecto a los involucrados del sexo femenino, no es posible establecer una relación concluyente entre DAP y sexo, pues cualquier afirmación estaría afectada por un sesgo muestral considerable. Se determinó la relación de la DAP con respecto a las variables utilizadas.

Para el caso de las variables ingreso, edad y nivel educativo, se obtuvo una relación positiva. En otras palabras, a medida que el individuo tiene mayor ingreso, está más dispuesto a pagar por el uso del 
agua; al igual que si el productor agrícola encuestado es de edad más avanzada, su disposición a pagar se incrementa; y entre mayor sea su nivel educativo, mayor será su disposición a pagar.

En el caso del precio, la relación es negativa, como se esperaba; si se incrementa el precio, los productores agrícolas encuestados estarán menos dispuestos a pagar. Si bien este resultado es un buen indicador, deben considerarse las limitaciones del estudio, ya que finalmente estos resultados muestran un aspecto subjetivo, en el sentido de que es una opinión; por tanto, se considera relevante la necesidad de profundizar en el estudio para determinar, entre otros aspectos: los costos reales del suministro del agua, si se corresponden con lo que pagan los usuarios; si existen pérdidas en el manejo del recurso; y conocer los impactos ambientales que genera el suministro. Por otro lado, hay que evaluar el aspecto social y considerar la relevancia del suministro de los alimentos, al margen de los costos que pueda significar el uso del agua.

\section{Anexos}

\section{Anexo 1. Cuestionario para la valoración contingente}




\section{Bloque 1. Preguntas de reconocimiento}

1. ¿Conoce la Presa Solís?

2. ¿Sabe de dónde proviene el agua que utiliza para el riego de sus cultivos?

3. ¿Sabe qué cantidad $\left(\mathrm{m}^{3}\right)$ de agua utiliza en sus cultivos?

4. ¿Conoce el costo del agua $\left(\mathrm{m}^{3}\right)$ para riego en la región?

5. ¿Conoce la disponibilidad de agua para riego en la región?

\section{Bloque 2. Preguntas de percepción}

6. ¿Considera que existe disponibilidad suficiente de agua para riego en la zona?

7. ¿Considera que la cantidad de agua que utiliza es alta o baja?

8. ¿Utiliza apropiadamente el agua, es decir, sólo la necesaria?

9. ¿Contabiliza el agua como insumo en la producción de sus cultivos?

10. ¿Cuál es la calidad de agua que utiliza en sus cultivos? 
11. ¿Podría usted costear su producción sin la utilización del agua de la presa Solís?

\section{Bloque 3. Disposición a pagar}

12. Tomando en cuenta lo anterior ¿Estaría dispuesto a pagar una cantidad representativa por la utilización del agua de la presa Solís?

a) Sí____ (Pase a la 14) b) No____ (Pase a la 13 y luego a la 16)

13. ¿Cuál es la principal razón por la que no estaría dispuesto a pagar?

14. ¿Qué cantidad estaría dispuesto a pagar por $\mathrm{m}^{3}$ de agua?

15. ¿Cuál es la principal razón por la que estaría dispuesto a pagar?

\section{Bloque 4. Datos socioeconómicos}

16. Género: Masculino Femenino 
17. Edad:

18. Estado civil: Soltero Casado

19. Nivel de estudios:

- Primaria

- Secundaria

- Preparatoria

- Licenciatura

- Posgrado

- Otro (especifique)

20. ¿Actualmente, cuál es su situación laboral principal?

a) Profesionista libre.

b) Profesor.

c) Comerciante.

d) Empleado.

e) Agricultor o ganadero.

f) Estudiante.

g) Ama de casa.

h) Otro (especificar).

21. ¿Cuál es el ingreso promedio mensual de su familia?

\begin{tabular}{|l|l|}
\hline Opción & Ingreso familiar mensual \\
\hline A & Menos de 2000 pesos \\
\hline
\end{tabular}




\begin{tabular}{|l|l|}
\hline B & De 2000 a 6000 pesos \\
\hline C & De 6000 a 8000 pesos \\
\hline D & De 8000 a 10000 pesos \\
\hline E & Más de 10000 pesos \\
\hline
\end{tabular}

22. ¿Número de miembros en la familia?

23. ¿Tipo de cultivo qué produce?

\section{Referencias}

Aguirre, R. (2015). Disposición a pagar por productos orgánicos en Chile: diferencias entre productos virtuosos y viciosos. Seminario para optar al título de Ingeniero Comercial. Seminario llevado a cabo en la Facultad de negocios. Santiago, Chile: Universidad de Chile.

FAO, Food and Agriculture Organization of the United Nation. (2015). Evaluación de recursos hídricos renovables. Revisión de la metodología de AQUASTAT 2015. Recuperado de http://www.fao.org/3/a-bc818s.pdf

Avilés, G., Soberanis, L., Troyo, E., Murillo, B., García, J., \& Beltrán, L. F. (2010). Valoración económica del servicio hidrológico del acuífero de la paz, B.C.S.: una valoración contingente del uso de agua municipal. Frontera Norte, 22(43), 103-128. 
Conagua, Comisión Nacional del Agua. (2016). Atlas del Agua en México 2016. http://201.116.60.25/publicaciones/AAM_2016.pdf

Del Ángel, P. A., Rebolledo, A., Villagómez, J., \& Zetina, R. (2009). Valoración del servicio ambiental hidrológico en el sector doméstico de San Andrés Tuxtla, Veracruz, México. Estudios Sociales (Hermosillo, Son.), 17(33), 225-257.

Flores, R., González, M., \& De los Santos, H. (2010). Valoración económica del servicio recreativo del Parque Hundido de la Ciudad de México. Región y Sociedad, 22(47), 123-144.

Jaramillo, L., Galindo, G., Bustamante, Á., \& Cervantes, J. (2013). Valoración económica del agua del río Tlapaneco en la "montaña de Guerrero", México. Tropical and Subtropical Agroecosystems, 16(3), 363-376.

Madurga, M. R. L. (2005). Los colores del agua, el agua virtual y los conflictos hídricos. Revista de la Real Academia de Ciencias Exactas, Físicas y Naturales de Madrid, 99, 369-389.

Medina J. (2014). La edad como factor del comportamiento del consumidor de productos orgánicos. Europan Scientific Journal, $10(7), 21-36$.

Novoa-Goicochea, Z. I. (2011). Valoración económica del patrimonio natural: las áreas naturales protegidas. Espacio y Desarrollo, (23), 131.

Salazar, S. (febrero, 2001). Conocimiento previo y disposición a pagar por mejoras ambientales. Encuentro de Economía Pública. 
Simposio llevado a cabo en el VIII Encuentro de Economía Pública, Universidad de Valencia, Valencia, España.

Sandoval, F., Valdivia, R., Cuevas, M., Hernández, J., Medellín, J., \& Hernández, A. (2016). Valoración económica del agua potable en la delegación Iztapalapa. Revista Mexicana de Ciencias Agrícolas, 7(6), 1467-1475.

Saucedo, G. J. (2015). Programa de gobierno municipal 2015-2018. Acámbaro, Guanajuato. Recuperado de https://portalsocial.guanajuato.gob.mx/documentos/programa-degobierno-de-ac\%C3\%A1mbaro-2015-2018

Semarnat, Secretaría de Medio Ambiente y Recursos Naturales. (2015). Estadísticas del agua en México. Edición 2015. México, DF, México: Comisión Nacional del Agua.

Silva, R., Pérez, G., \& Návar, J. (2010). Valoración económica de los servicios ambientales hidrológicos en El Salto, Pueblo Nuevo, Durango. Madera y Bosques, 16(1), 31-49.

Trujillo, J., Hernández, J., \& Martínez, M. (2019). Disposición a pagar por productos orgánicos en Texcoco, Estado de México. Revista Mexicana de Ciencias Agrícolas, 10(7), 1685-1691.

Tudela, J. (2011). Valoración económica de los beneficios de un programa de recuperación y conservación en el Parque Nacional Molino de Flores, México. Revista Chapingo Serie Ciencias Forestales y del Ambiente, 17(2), 231-244. 
Tudela, J. (2017). Estimación de beneficios económicos por el mejoramiento del sistema de tratamiento de aguas residuales en la ciudad de Puno, Perú. Revista Desarrollo y Sociedad, 79, 189.

Valdivia, R., García, E., López, M., Hernández, J., \& Rojano, A. (2011). Valoración económica por la rehabilitación del río Axtla, SLP. Revista Chapingo Serie Ciencias Forestales y del Ambiente, 17(3), 333-342.

Vázquez, P. (2012). Percepción del consumidor y productor de orgánicos: el mercado Ocelotl de Xalapa, Ver. México. Revista Mexicana de Agronegocio, 16(31), 20-29.

WWC, World Water Council. (2015). World Water Council 2016-2018 Triennial Strategy. Marsella, France: World Water Council. Recuperado de http://www.worldwatercouncil.org/es/publications 